und Lageverhăltnis eigentümlieher an der Schleimhaut des mensehlichen Mittelohres vorkommender Organe." Wien 1870. - Striekers Handbuch der Gewebelebre: 1870 "Das ăuBere und mittlere 0 hr." - K. K. Akademie der Wissenseh. Bd. 66, 1872: "Versuche über die Akkomodation des Ohres" von E. Mach u. J. Kessel. - Ebda. Bd. 66: "Die Funktion der Trommelhöhle und der Tuba Eustachii" von E. Mach u. J. Kessel. - Ebda. Bd. 69, 1874: "Beiträge zur Topographie und Mechanik des Mittelohres" von E, Maeh" u. J. Kessel. - A. f. O. Bd. VIII, 1873: "Über den EinfluB der Binnenmuskeln der Paukenhöhle auf die Bewegungen und Schwingungen am toten Ohre." - A. f. O. Bd. XI, 1876: Über die Durehsehneidung des Steigbügelmuskels beim Menschen und über die Extraktion des Steigbügels, resp. der Columella bei Tieren". - Wien. mediz. Presse, 1878, Nr. 51: . Über die Bedeutung der Erkrankung des Nasenrachenraumes. “ - Osterr. Arztl. Vereinszeitung 1879: "Über das Aussehneiden des Trommelfelles und Mobilisieren des Steigbügels." - A. f. O. Bd. 18: 1. -Über die Funktion der Ohrmuschel bei den Raumwahrnehmungen." 2. "Über die Verschiedenheit der Intensität eines linearerregten Schalles in den verschiedenen Kichtungen." 3. "Über das Hören von Tönen u. Geräuschen." - Correspondenz-Blätter d. Allgem. ärztl. Vereins f. Thüringen 1887: "Über die Behandlung d. chron. eitrigen Mittelohrentzündung." - Ebda. 1888: "Über die chron. Katarrhe d. Mittelohres u. ihre Behandlung." - Ebda. 1889: "Über die Exostosen des äußeren Gehörganges." - Ebda. 1890: "Uber die Fremdkörper im Ohre." - A. f. 0. 1890 (Bd. 31): "Über die vordere Tenotomie.“ - Cordespond.-Bl. d. ărztl. Vereins f. Thüringen 1891: „Einiges über die Bedeutung und die Untersuchungsmethoden der Nasenhöhle und des Nasenrachenraumes." Schwartze's Handbuch der Ohrenheillunde I. Bd.: "Die Histologie der Ohrmuschel, des äußeren Gehörganges, Trommelfells und Mittelohres." "Uber die vordere Tenotomie, Mobilisierung und Extraktion d. Steigbügels." Verlag von G. Fischer, Jena. 1594. (Referat A. f. O. Bd. 42, S. 57.) A. f. 0. Bd. 57, 8. 177: Rede zur feierlichen Eröffnung der neuen Universitäts-Ohrenklinik in Jena am 14. Dezember 1900.

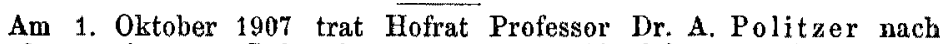
Vollendung seines 72 . Lebensjahres und nach Absolvierung seines akademischen Ehrenjabres als Lehrer der Ohrenheilkunde, den österreichischer Universitätsgesetzen entsprechend, von der Leitung der Klinik für Otiatrie zurück.

Die für diesen Tag von einem internationalen Komitee geplant gewesene große akademische Feier mußte auf ausdrücklichen Wunsch Politzer unterbleiben; seinen Intentionen entsprechend erfolgte nur die Überreichung der von Teles (Budapest) ausgeführten Plaquette Politzers und die Übergabe einer Adresse mit den Namen aller an der Ehrung Politzers sich beteiligenden Kollegen und Schüler in allen Weltteilen.

In dem mit Blumen festlich geschmückten Hörsaale der Ohrenklinik waren zur Feier erschienen: Vertreter des Unterrichts-Ministeriums und der Statthalterei, die meisten Professoren und Privatdozenten der Wiener medizinischen Fakultăt, der Direktor und viele Primarărzte des $\mathbf{K}$. K. allgemeinen Krankenhauses, zahlreiche Freunde, Schüler und Verehrer Politzers, alle Herren der Ohrenklinik und alle früheren und aktiven Assistenten der Wiener Ohrenklinik.

Die bei dieser Feier gehaltenen Ansprachen und die Abschiedsrede des Gefeierten sollen ausführlich später mitgeteilt werden. Sc hwartze.

\title{
Berichtigung zu Band 72.
}

S. 14, Zeile 8 von oben, muß es heißen statt "direktem" indirektem. S. 16, Zeile 3 von unten, muB es heißen $1891-1905$ statt $1891-1895$. S. 17 , Zeile 9 von oben, mub es heißen 1905 (1. 10.) - 1907 (15. 2.) statt 1905 (10. bis 15.2 .) -1907 . 